\title{
IN SEARCH OF IDEAL KNOTS*
}

\author{
PIOTR PIERAŃSKI \\ Department of Physics, Poznań University of Technology, \\ Institute of Molecular Physics, Polish Academy of Sciences \\ e-mail: pieransk@man.poznan.pl
}

\begin{abstract}
A concise introduction to the mathematical theory of knots is presented. Definitions of basic notions are explained. Then, knots are considered as material objects and the question concerning their optimal conformations is posed. A numerical algorithm searching for the ideal knots is described. A few results obtained with its help are presented.
\end{abstract}

\section{INTRODUCTION}

Let us start with a few formal definitions of the basic notions of the topological theory of knots [1],

\section{Knot}

Knot is a self-avoiding, closed curve in $3 D$ space.

For mathematicians, knots are thus tied on something immaterial - an inifnitely thin thread, neccesserily closed at the end of the tying procedure. The length $L$ of the thread is of no significance - a knot of any shape can be scaled up or down without any hindrance.

Fig. 1. A knot tied on a tube of a finite thickness

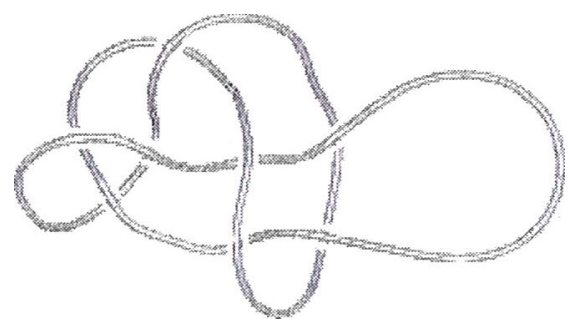

Physicists, however, imagine knots as material objects. If a knot is tied on a string or a tube (let us remember: neccesserily closed at the end of the tying procedure), the condition of self-avoidance found within the formal definition is surely fulfilled. Figure 1 shows a knot tied on a tube. If the diameter $D$ of the tube is kept fixed, the knot cannot be scaled down as much as one wishes. There is a certain, minimal size of the knot and connected with it minimal length $L$ at which the finite diameter of the tube will create problems. We shall return to the problem later. The 3D self-avoiding curve forming a given knot can be deformed. From the topologial point of view all deformations, during which the condition of self-avoidance has been not violated, do not matter; under such deformations the knot changes its conformation but keeps identity. This is stated in the second definition:

* Lecture delivered in a series of seminars Computational methods in science and technology 


\section{Knot type}

Two knots are of the same type if they can be transformed into each other via deformations during which the knots remain self-avoiding.

The 3-dimensional transformations which fulfill the criterion of keeping a knot selfavoiding are called ambient isotopy. Figure 2 shows a sequence of frames taken from a simulation of a continuous deformation process in which the knot shown in the first figure has been transformed into a circle. This numerical experiment proves that the knot type of the initial knot $K_{I}$ is identical with the knot type represented by the final knot $K_{2}$.
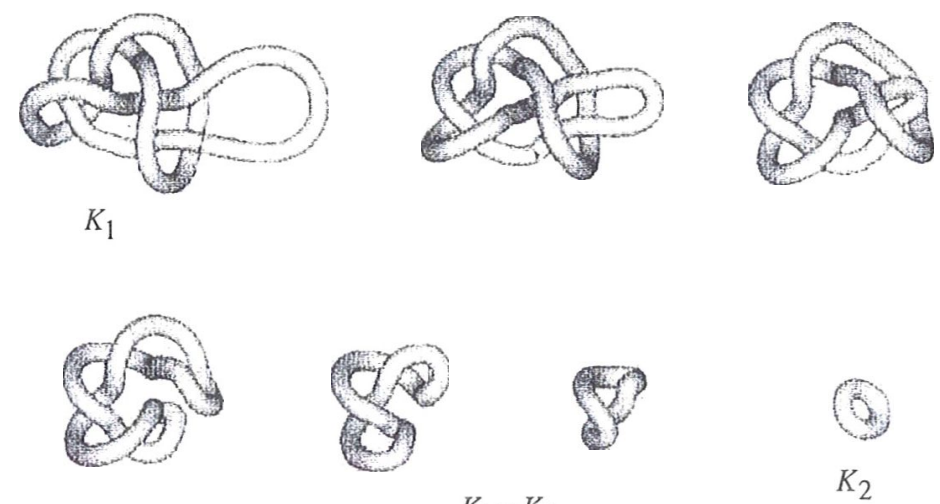

$$
K_{1}=K_{2}
$$

Fig. 2. A continuous deformation process during which the knot remains selfavoiding. Its existence proves that knots $K_{1}$ and $K_{2}$ are of the same type

Most often knots cannot be transformed into each other - they are of a different knot types. How many different knot types are there? The answer is: infinitely many. Figure 3 presents three most simple knot types.

As easy to check, tying two nontrivial knots on the same piece of rope creates a new knot, which is not simpler than the factor knots; they never anihilate each other to result in a trivial knot. Given a knot, one should check, if it is not composed of two or more simpler knots. We need here another definition:
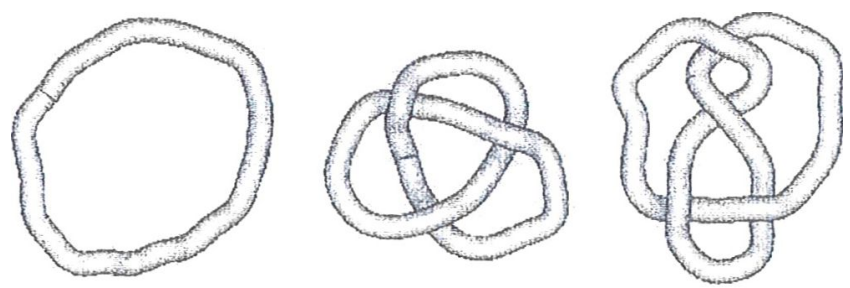

Fig. 3. The simplest knot types 


\section{Composite knots}

Knots, which can be seen as two nontrivial knots connected but with two pieces of a rope are called composite.

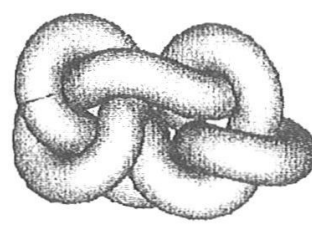

$K=K_{1} \# K_{2}$

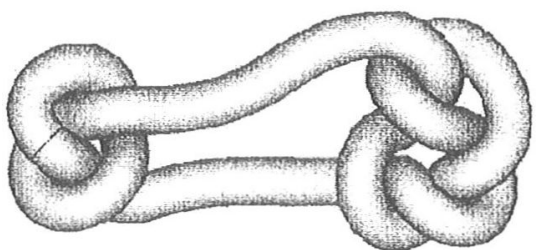

$K_{1}$

$K_{2}$

Fig. 4. An example of a composite knot. As shown in the ight part pf the figure the knot can be seen as a composition of two nontrivial knots: a trefoil knot and a figure eight knot

This brings us to the definition of:

\section{Prime knots}

Knots, which are not a composition of any two nontrivial knot, are called prime.

Having defined prime knots we may ask a basic and by no means simple question:

How to classify and tabulate prime knots?

Looking at a knot from a given direction, or projecting it on a plane, we see it as a two-dimensional curve, which (except the trivial knot) crosses itself in a few points.

Deforming the knot via ambient isotopy transformations (i.e. via transformations during which the curve remains self-avoiding in the 3-dimensinal space) and looking at it from different directions we shall find that the number of crossings changes. It cannot be reduced though below a certain, well defined for each knot minimal number of crossings. To illustrate this, we repeat below Figure 2 indicating this time how during the ambient isotopy transformation performed on the knot its crossing number becomes reduced from 8 to 0 .

Fig. 5. In the projection on a plane the knot is seen as a 2-dimensional curve
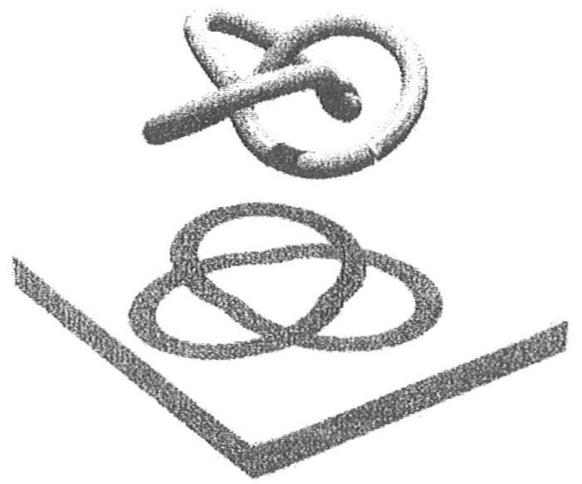


\section{Crossing number $(\mathrm{CN})$}

$C N$ - number of crossings seen in a projection of a knot onto a plane.

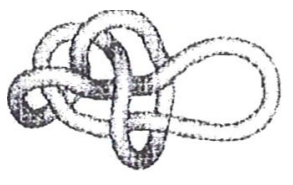

$\mathrm{CN}=8$

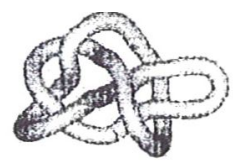

$\mathrm{CN}=7$

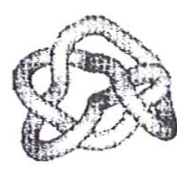

$\mathrm{CN}=7$
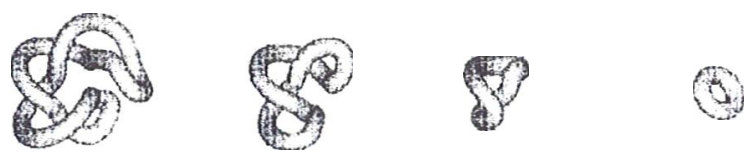

$\mathrm{CN}=3$

$\mathrm{CN}=2$

$\mathrm{CN}=1$

$\mathrm{CN}=0$

Fig. 6. A few frames from a transformation via which the number of crossings seen a knot has been reduced to 0

\section{Minimal crossing number (MCN)}

MCN - the minimal number of crossings possible to be seen in a projection of a knot onto a plane

Knots can be segregated into classes according to their minimal crossing number. This is the basic criterion of the tabulation of knots. Its fascinating history is described by Jozef Przytycki [2], one of a few Polish mathematicians, whose names are found in all textbooks on the knot theory. Let me cite here a few essential names and dates.

1880 - Reverend Thomas Penyngton Kirkman - the first attempt to tabulate knots.

1877 - Peter Guthrie Tait (a Scottish physicist) - tabulation of all alternating (see below) knots up to $M C N=10$.

1899 - Charles Newton Little - tabulation of all prime knots (including nonalternating, see below) up to $M C N=10$. His table was discovered to contain one duplication (see Perko, below). An attempt to tabulate knots with $M C N=11$. It was found later that the table contained 11 omissions and one duplication.

1917 - Mary G. Haseman - a complete table of all amphicheiral (see below) knots with up to $M C N=12$.

1927 - J. Alexander and Briggs - the first rigorous proof that most of the tabulated knots with up to $M C N=9$ were distinct.

1932 - Kurt Reidemeister - finishing the work initiated by Alexander and Briggs.

1969 - John H. Conway - invention of a new notation for knots and based on it tabulation of all prime knots up to $M C N=11$, not without errors, however. 
1974 - Kenneth A. Perko (a New York lawyer) - discovery of one duplication in the Little table and four omissions in the Conway table.

1978 - Alain Caudron - the first correct table of all prime knots up to $M C N=11$.

1983 - Hugh Dowker - invention of another notation for knots.

Morwen Thistlethwait - a numerical algorithm generating knots and created with it tabulation of all prime knots up to $M C N=13$.

The table below presents the numbers $N$ of prime knots tabulated by Thislethwait found in their classes with $M C N$ from 0 to 13.

Table I. Number $N$ of prime knots versus their minimal crossing number

\begin{tabular}{|c|c|c|c|c|c|c|c|c|c|c|c|c|c|c|}
\hline$M C N$ & 0 & 1 & 2 & 3 & 4 & 5 & 6 & 7 & 8 & 9 & 10 & 11 & 12 & 13 \\
\hline$N$ & 1 & 0 & 0 & 1 & 1 & 2 & 3 & 7 & 21 & 49 & 165 & 552 & 2176 & 9988 \\
\hline
\end{tabular}

As seen in the table, number $\mathrm{N}$ of knots grows rapidly with MCN. How rapidly? It was shown in 1987 by Claus Ernst and Dewitt Sumners (who used results obtained by Kauffman, Murasugi and Thistlethwait) that $\mathrm{N}$ is not smaller than:

$$
\left(2^{\text {MCN-2}}-1\right) / 3
$$

That classes of prime knots with $M C N-1$ and 2 are empty one can check on one's own. See Figure 7.
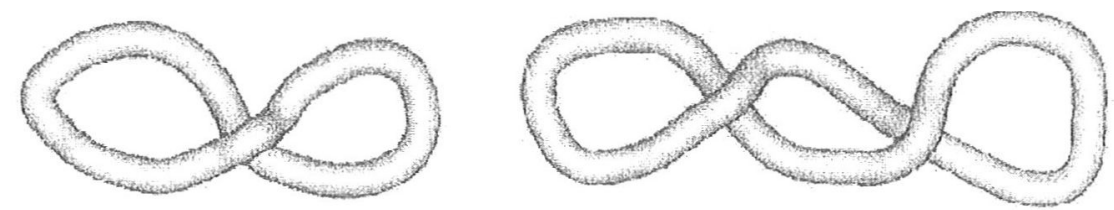

Fig. 7. Examples of knots with $C N=1$ and $C N=2$. It is obvious that by a simple manipulation the crossings can be removed

Talking about different knots is possible only if one is able to name them. Such names as "trivial", "trefoil" or "figure eight" are customary, but most often we use one of a few more concise notations. For instance, according to one of the notations, knots are identified by two numbers: $n_{\mathrm{m}}$.

$n$ - describes here the class within which the knot has been tabulated, i. e. its minimal crossing number, thus, $n=M C N$,

$m$ - describes position of the knot within the class specified by $n$ in table set up by Rolfsen.

In this notation trivial knot is described as $0_{1}$, trefoil knot as $3_{1}$ and figure eight knot as $4_{1}$. 


\section{Cheiral and amphicheiral (chiral and achiral) knots}

Let us take

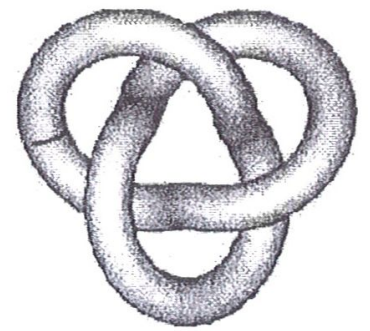

the $3_{1}$ knot,

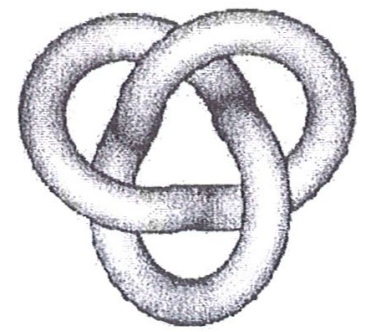

its mirror image

and ask the question:

Are the two knots ambient isotopic?

Answer, not easy to find though, is:

No.

For obvious reasons in the table of knots only one of the knots is tabulated. Similarity, let us take

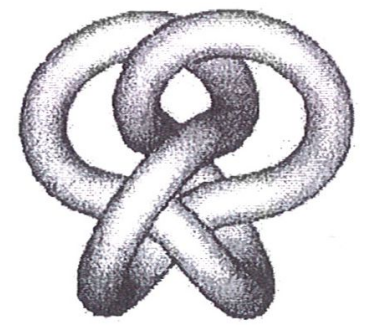

the $4_{1}$ knot,

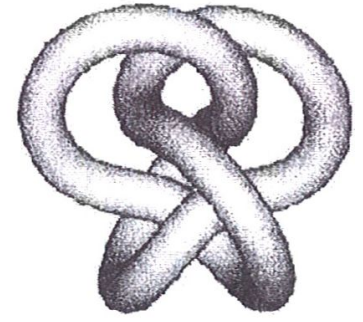

its mirror image

and ask the same:

Are the two knots ambient isotopic?

Answer, possible to find by a simple manipulation of the string on which the knot has been tied is this time:

Yes.

We say that $3_{1}$ knot is cheiral (or chiral) and $4_{1}$ knot is amphicheiral (or achiral).

Looking at a particular conformation of a knot from different directions one can find out that the number of crossings changes. (If the knot conformation is a suitable one, one may find for it a direction from which the minimal crossing number is seen). One may ask, which is the average number of crossings. 


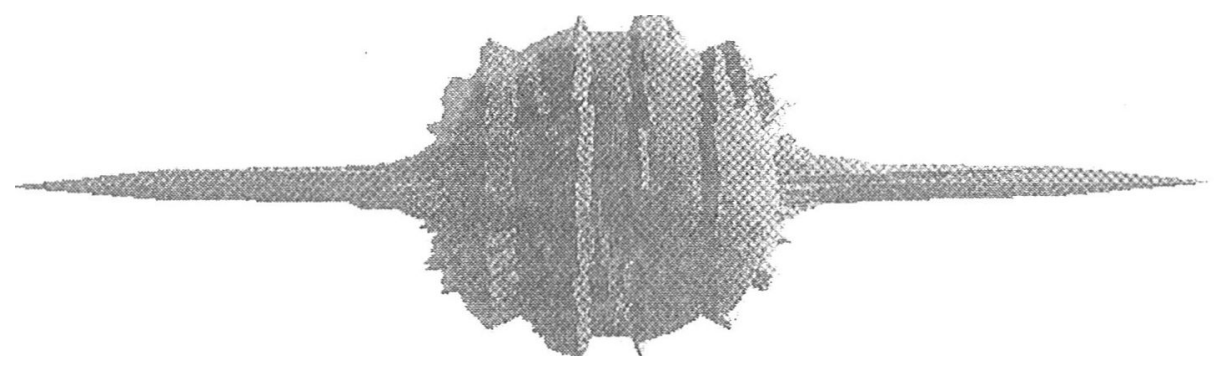

Fig. 8. A map of the crossing number for the $11_{1}$ knot

\section{Average crossing number ( $\mathrm{ACN})$}

$A C N$ - the average number of crossings seen within the knotfrom all possible directions.

$$
A C N=\frac{1}{4 \pi} \int_{-\pi}^{\pi} \int_{0}^{2 \pi} C N(\Theta, \varphi) \cos \Theta d \Theta d \varphi
$$

where $C N(\Theta, \varphi)$ is the crossing number as seen in the projection determined by angles $\Theta$ and $\varphi$

The formula presented above is of not much use. How to implement it for a knot $K$ given e. g. in an analytical form? To calculate $A C N$ one should find first the $C N(\Theta, \varphi)$ function. It is by no means clear, if this is possible at all. Fortunately, $A C N$ can be calculated in a much more smart manner, using another double integral [3]:

$$
A C N=\frac{1}{4 \pi} \oint_{K K} \frac{\left|\left(d \vec{r}_{1} \times d \vec{r}_{2}\right) \cdot \vec{r}_{1,2}\right|}{r_{1.2}^{3}} .
$$

Analysing crossings seen in a knot $K$ from a given direction one may get into more detailed analysis. First, let us orient it e. g. by imagining that some objects (1-dimensional cars, for instance) move along it (using $K$ as a one way street). Looking at a knot from the given direction we shall see the crossings as bridges. If the drivers moving on the bridges see the cars on the street below heading left, the crossing is labeled with +1 , otherwise it is labeled -1 . A sum of the absolute values of the labels gives of course the directional crossing number $C N$; a sum $W$ of the signed values of the labels gives another important variable used in the theory of knots: the directional writhe or simply the writhe of $K$ [4].

The directional writhe is an integer. Its absolute value is not larger then the directional crossing number $C N$. Let us note that a change in the orientation of the knot does not change the sign of $W$.

As in the case of the crossing number, the directional writhe can be averaged over all possible directions. The average is called the writhing number of $K$ or simply its writhe. Below we shall denote it by $W r$. (Unfortunately, the brief name of the writhing number coincides with the brief name of the directional writhe. Which of them is referred to becomes usually clear from the context.) Its value can be calculated with the use of an integral similar to that used in the calculation of the average crossing number: 


$$
W r=\frac{1}{4 \pi} \oint_{K K} \oint_{\frac{\left(d \vec{r}_{1} \times d \vec{r}_{2}\right) \cdot \vec{r}_{1,2}}{r_{1,2}^{3}}} .
$$

REMARK. There exists an interesting connection of writhe with the Berry phase or the Hannay angle, a connection which seems to be not well explored yet.

\section{IDEAL KNOTS}

For a topologist all conformations of a knot are just equivalent. For a physicist, who imagines a knot as a material object, different conformations of a knot may be not equivalent. In particular, when one considers a knot as tied on a perfectly flexible, but at the same time perfectly hard in its circular crossection tube, one may ask a question:

Which is the tightest conformation of a knot, i.e. which is the conformation of the knot possible to be tied on the shortest piece of the tube.

The question was asked by a team of scientists lead by A. Stasiak in a paper published in Nature [5],

To find the "ideal", as they were called by Stasiak, conformations, Vsevolod Katritch used the numerical method of the simulated annealing. Using the method, Katritch managed to find apparently most tight conformations of a few simple and a few composite knots.

Stimulated by the work of the Lausanne team I developed SONO (shrink-on-no-overlaps), a simple algorithm able to tighten knots tied on a tube [6],

In the algorithm the knot is discretised: it consists of a number $N$ of equidistant nodes. The nodes repel each other with a hard sphere potential of the diameter $D$. For obvious reasons, the neighbouring nodes do not interact.

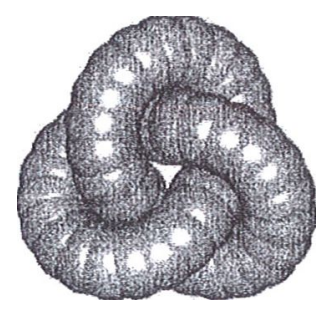

a)

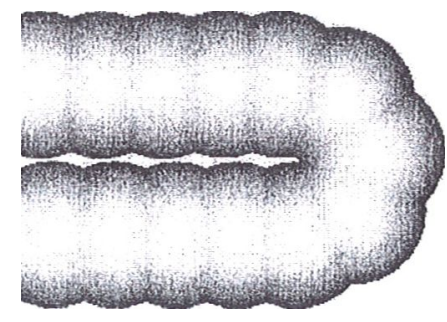

b)

Fig. 9. (a) - the discrete representation of the trefoil knot, (b) - the most tight turn

The idea of the algorithm is as follows: remove all overlaps between the spheres surrounding each of the $N$ nodes, having done this - shrink the conformation [7]. Removing the overlaps, one must simultaneously keep an eye on the distances between the neighbouring nodes. One may imagine that the nodes are kept together by a set of $N$ leashes, all of the same length. The Pascal implementation of the SONO is built from a set of procedures. The names of the basic ones explain their function: 


\section{ControlLeashes (CL) \\ FindNeighbours (FN) \\ RemoveOverlaps (RO).}

\section{TESTS OF THE SONO ALGORITHM}

One of the most obvious features of any algorithm which searches for the most tight conformation of knots should be its ability to untangle the arbitrarily entangled conformation of the trivial knot. (Its most tight conformation - a torus - is obvious). Figure 10.

The second test was suggested by Moffatt [8]: "It would be interesting to test the algorithm on the simpler $\mathrm{T}_{3,2}$ and $\mathrm{T}_{2,3}$ configurations of the trefoil; it is not clear to me, how $\mathrm{T}_{3,2}$ could flow to $T_{2,3}$ through the process described". Figure 11 presents the flow of the $(3,2)$ torus knot towards its most tight form. Note the transition in the symmetry of the conformation during its tightening: the initial one has a twofold symmetry axis, while the final one the symmetry axis is the threefold

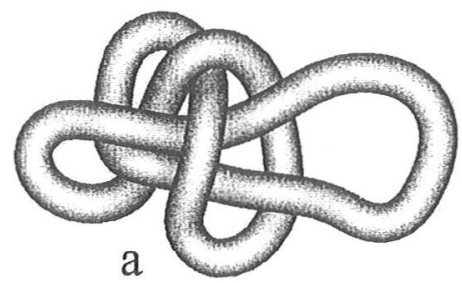

a

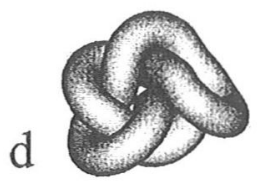

(a)

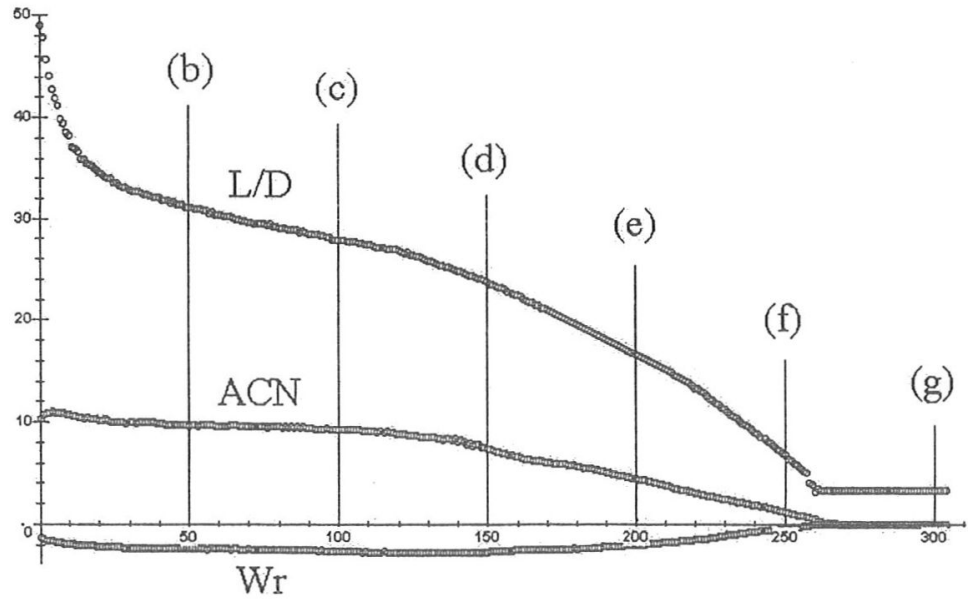

Fig. 10. Evolution of the conformation of the initially entangled trivial knot under the action of the SONO algorithm. The plot below shows the evolution of the $L I D, A C N$ and $W r$ parameters 
As mentioned before, tabulation of knots is not a simple problem. In 1974 Kenneth A. Perko, a New York lawyer discovered of a duplication in the Little table of knots with 9 crossings. SONO algorithm reveals the duplication in a few minutes. Figure 12.

Searching for the ideal conformation of even very simple knots is not easy. Soon after Ref. 4. has been published, Katritch and I discovered, that some of the "ideal" conformations shown in the paper were not the most tight ones. For instance, the conformation of the $5_{1}$ knot shown in the paper has clearly a fivefold symmetry axis. A simulation performed with the use of the SONO algorithm proved that the symmetry becomes broken. Figure 13.

A much more spectacular example of the symmetry breaking process is shown in Figure 14. Here, not only the symmetry becomes broken, but the structure of the knot undergoes a profound reconstruction. As discovered in more recent simulations, conformation (f) is not the best one.

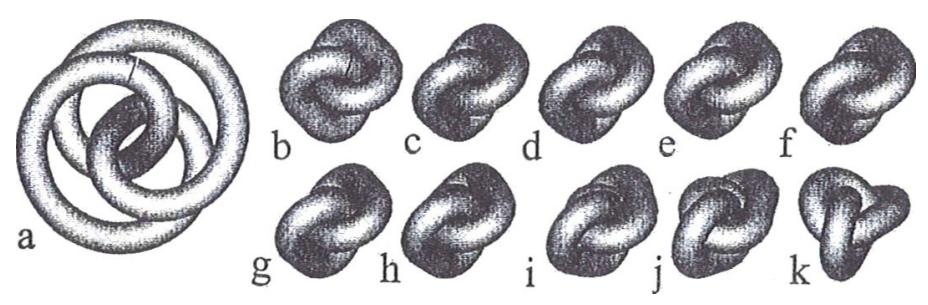

Fig. 11. Evolution of the $(3,2)$ torus knot towards its $(2,3)$ ideal form

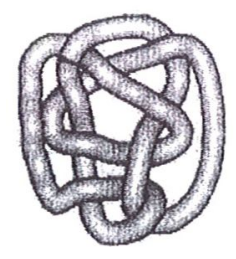

a)

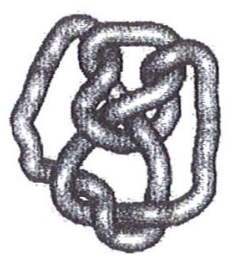

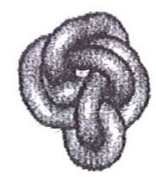

b)

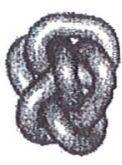

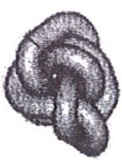

c)

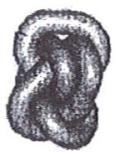

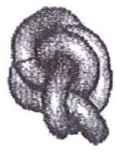

d)

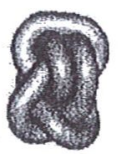

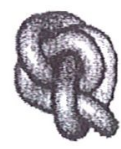

e)

Fig. 12. Induced by the SONO algorithm evolution of knots from the Pairko pair towards a single conformation

The case of the $55_{1}$ knot proves that during the tightening process knots may enter conformations which are not the ideal one. To get out of such local minima is not easy. SONO algorithm is able to do it in some cases, in others - not. The composite knots are apparently the most difficult ones. 


\section{RESULTS}

In a close cooperation with the Lausanne group I performed a long series of simulations looking for the most tight conformations of all simple knots with up to 9 crossings and some of the composite knots. Parameters of the most tight conformations I managed to find are shown in Table II. A plot of the average crossing number $A C N$ of the knots versus their length $L / D$ proves that the linear correlation between the two parameters suggested in Ref. 5 is but the first approximation. Most probably the relation has a character of a power law. Figure 16 and Figure 17.

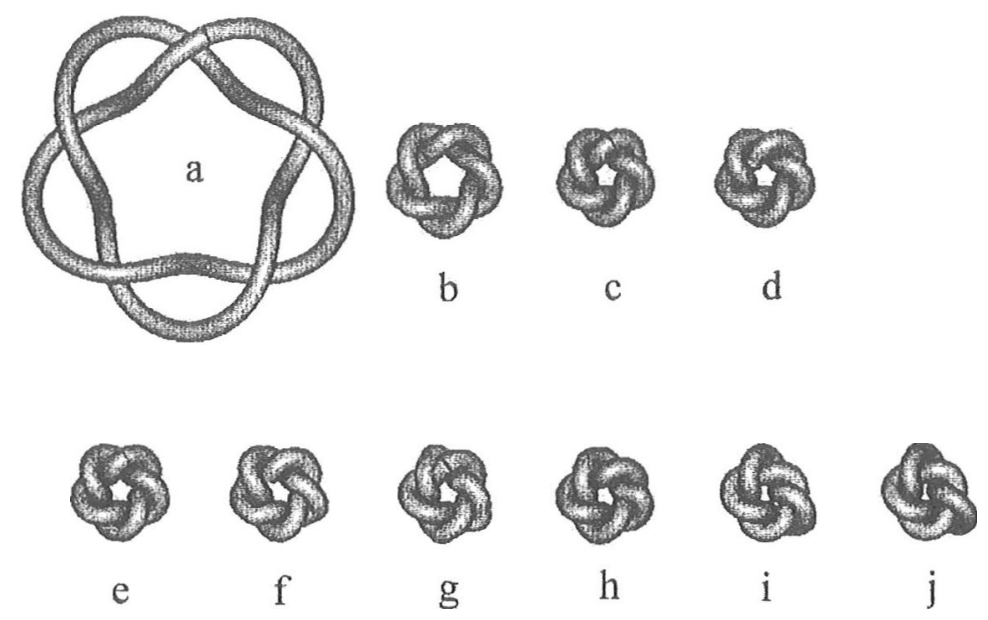

Fig. 13. The symmetry breaking observed during the tightening of the $(2,5)$ torus knot during its tightening forced by the SONO algorithm
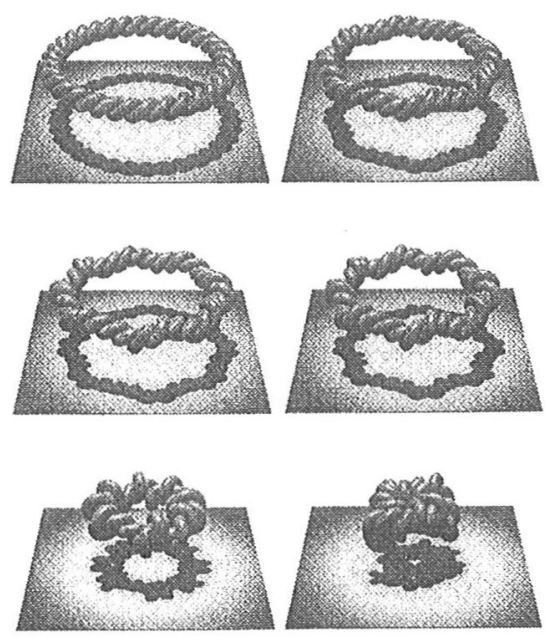

Fig. 14. Symmetry breaking observed in the $(2,33)$ torus knot. The tube on which the knot was tied is shown at half of its actual diameter 

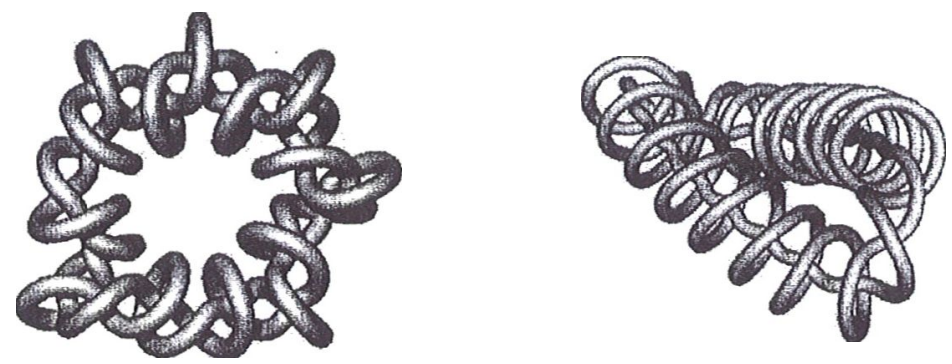

Fig. 15. Two tight conformations of the $(2,33)$ torus knot. The conformation shown on the right proves to be a better one

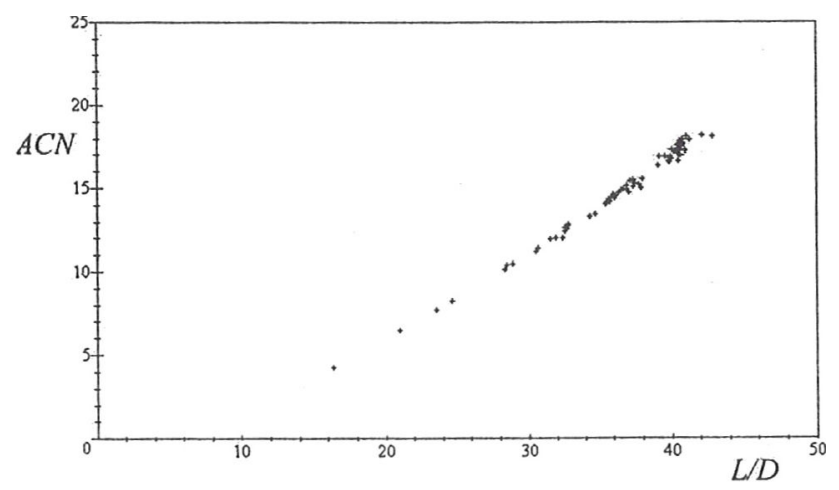

Fig. 16. $A C N$ vs $L I D$ for all simple knots with up to 9 crossings

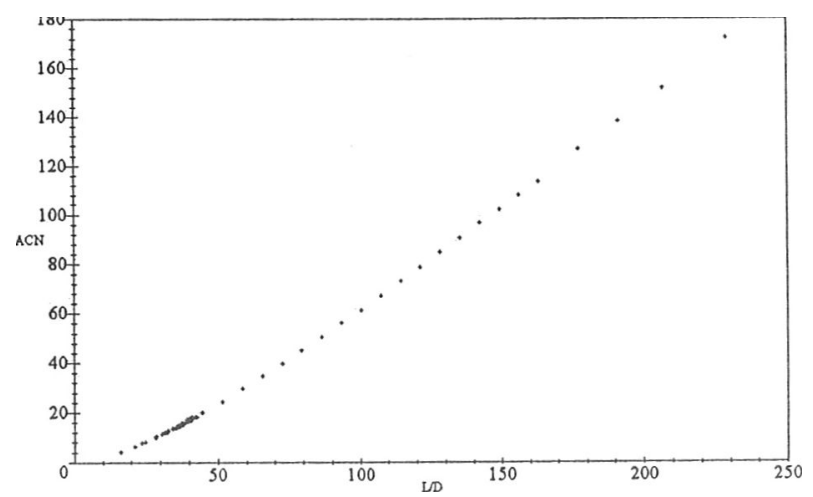

Fig. 17. $A C N$ vs $L I D$ for all simple knots with up to 9 crossings and number of larger knots from the family $(2, n)$ of the torus knots

In contrast to the $A C N$ vs $L / D$ dependence, the linear writhe vs. minimal crossing number correlations suggested in Ref. 5 prove to be well supported by may data. Figure 18. The correlations seem to be very interesting and are intensively studied in the cooperation with A. Stasiak.

Properties of the tight conformations of the composite knots are very interesting as well. For instance, their writhe proves to be additive, i. e. the writhe value of the composite knot is, (with an unexpected accuracy) equal to the sum of the writhe of the factor knots [9]. 


\section{CONCLUSIONS}

The development of the theory of knots enters a new phase. Within the society of the mathematicians knots were so far interesting to the topologists - now also the geometry, in particular,

Table II. Parameters of the most tight conformations found with the SONO algorithm

\begin{tabular}{|c|c|c|c|c|c|c|c|}
\hline TYPE & $L / D$ & $A C N$ & $W r$ & & & & \\
\hline $3_{1}$ & 16.33 & 4.26 & 3.39 & $9_{8}$ & 40.19 & 17.16 & 2.24 \\
\hline $4_{1}$ & 20.99 & 6.47 & 0.00 & 99 & 39.90 & 16.60 & 10.23 \\
\hline $5_{1}$ & 23.55 & 7.75 & 6.25 & $9_{10}$ & 39.82 & 16.61 & 8.56 \\
\hline $5_{2}$ & 24.68 & 8.21 & 4.56 & $9_{11}$ & 40.63 & 16.87 & 6.16 \\
\hline $6_{1}$ & 28.30 & 10.15 & 1.13 & $9_{12}$ & 40.01 & 16.88 & 4.52 \\
\hline $6_{2}$ & 28.47 & 10.39 & 2.77 & $9_{13}$ & 40.31 & 16.94 & 8.57 \\
\hline $6_{3}$ & 28.88 & 10.52 & 0.02 & $9_{14}$ & 39.98 & 16.93 & 1.79 \\
\hline $7_{1}$ & 30.70 & 11.36 & 9.10 & $9_{15}^{14}$ & 41.00 & 17.32 & 4.53 \\
\hline $7_{2}$ & 32.41 & 12.18 & 5.74 & $9_{16}$ & 40.00 & 16.74 & 10.17 \\
\hline 73 & 31.90 & 12.07 & 7.40 & $9_{17}$ & 40.15 & 17.19 & 2.20 \\
\hline $7_{4}$ & 32.53 & 12.61 & 5.82 & $9_{18}$ & 40.72 & 17.50 & 8.56 \\
\hline 75 & 32.57 & 12.38 & 7.43 & $9_{19}$ & 41.01 & 17.29 & 0.67 \\
\hline $7_{6}$ & 32.82 & 12.76 & 3.43 & $9_{20}$ & 42.86 & 18.00 & 6.53 \\
\hline $7_{7}$ & 32.76 & 12.80 & 0.66 & $9_{21}$ & 40.49 & 16.88 & 4.50 \\
\hline $8_{1}$ & 35.46 & 14.02 & 2.29 & $9_{22}$ & 40.44 & 17.41 & 2.14 \\
\hline $8_{2}$ & 35.63 & 14.30 & 5.63 & $9_{23}$ & 40.57 & 17.48 & 8.54 \\
\hline $8_{3}$ & 35.50 & 14.00 & 0.00 & $9_{24}$ & 40.42 & 17.06 & 0.58 \\
\hline $8_{4}$ & 35.93 & 14.57 & 1.62 & $9_{25}$ & 40.51 & 17.64 & 4.57 \\
\hline $8_{5}$ & 36.01 & 14.60 & 5.60 & $9_{26}$ & 40.36 & 17.14 & 3.48 \\
\hline $8_{6}$ & 36.15 & 14.50 & 3.97 & $9_{27}$ & 40.81 & 17.71 & 0.63 \\
\hline $8_{7}$ & 36.04 & 14.42 & 2.83 & $9_{28}$ & 40.71 & 17.54 & 3.40 \\
\hline $8_{8}$ & 36.58 & 14.79 & 1.19 & $9_{29}^{-0}$ & 40.71 & 17.80 & 2.23 \\
\hline $8_{9}$ & 36.17 & 14.52 & 0.04 & $9_{30}$ & 40.88 & 17.75 & 0.60 \\
\hline $8_{10}$ & 36.63 & 14.93 & 2.78 & $9_{31}$ & 40.74 & 17.83 & 3.37 \\
\hline $8_{11}$ & 38.02 & 15.49 & 3.88 & $9_{32}$ & 40.68 & 17.58 & 3.46 \\
\hline $8_{12}$ & 36.94 & 14.91 & 0.00 & $9_{33}$ & 41.34 & 17.98 & 0.61 \\
\hline $8_{13}$ & 36.35 & 14.80 & 1.21 & $9_{34}$ & 41.02 & 18.06 & 0.59 \\
\hline $8_{14}$ & 36.89 & 15.03 & 4.03 & $9_{35}$ & 40.05 & 17.29 & 6.99 \\
\hline $8_{15}$ & 37.11 & 15.38 & 7.91 & $9_{36}$ & 40.42 & 17.60 & 6.23 \\
\hline $8_{16}$ & 37.39 & 15.42 & 2.76 & $9_{37}$ & 40.68 & 17.42 & 0.54 \\
\hline $8_{17}$ & 37.19 & 15.41 & 0.01 & $9_{38}$ & 40.89 & 17.93 & 8.58 \\
\hline $8_{18}$ & 37.40 & 15.50 & 0.08 & $9_{39}$ & 42.19 & 18.09 & 4.61 \\
\hline $8_{19}$ & 30.46 & 11.20 & 8.64 & $9_{40}$ & 40.77 & 17.67 & 3.42 \\
\hline $8_{20}$ & 31.56 & 11.96 & 1.96 & $9_{41}$ & 40.63 & 17.76 & 1.82 \\
\hline $8_{21}$ & 32.72 & 12.61 & 4.66 & $9_{42}$ & 34.73 & 13.15 & 1.18 \\
\hline 9 & 37.81 & 15.18 & 11.99 & $9_{43}$ & 35.78 & 14.24 & 5.23 \\
\hline $9_{2}$ & 39.63 & 16.90 & 6.86 & $9_{44}$ & 35.79 & 14.48 & 1.32 \\
\hline 93 & 39.20 & 16.82 & 10.25 & $9_{45}^{44}$ & 37.41 & 15.18 & 5.18 \\
\hline $9_{4}$ & 39.11 & 16.32 & 8.52 & $9_{46}$ & 34.27 & 13.55 & 2.57 \\
\hline $9_{5}$ & 39.78 & 16.85 & 6.95 & $9_{47}$ & 37.49 & 15.25 & 2.78 \\
\hline $9_{6}$ & 39.96 & 16.89 & 10.25 & $9_{48}$ & 37.06 & 14.73 & 3.95 \\
\hline $9_{7}$ & 40.22 & 16.49 & 8.56 & $9_{49}$ & 36.91 & 14.99 & 7.90 \\
\hline
\end{tabular}




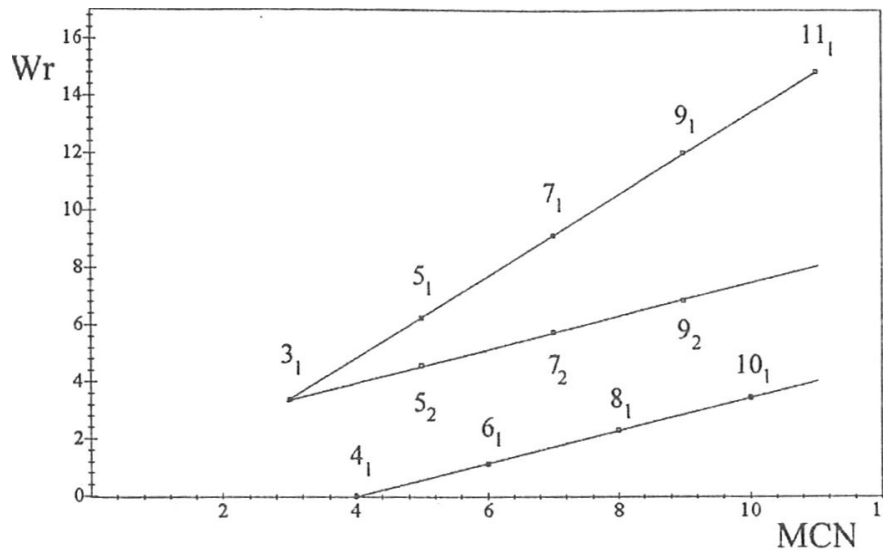

Fig. 18. Writhe vs minimal crossing number for a few families of simple knots

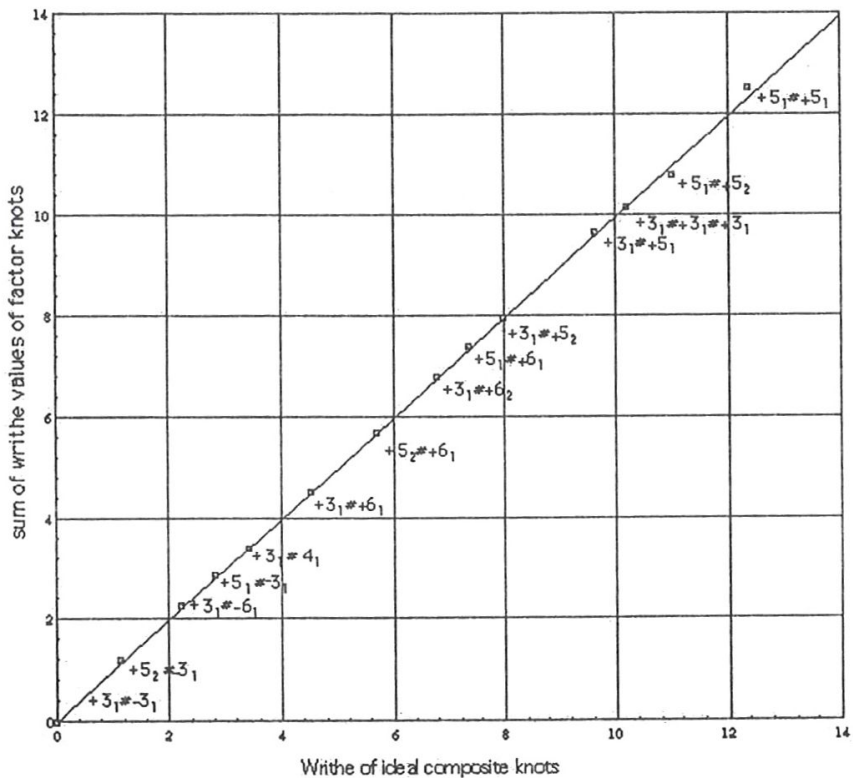

Fig. 19. The writhe of the tight conformations of composite knots versus the sum of the writhe of their factor knots

the differential geometry becomes interested in properties of their various conformations. Within the society of the physicist, knots were treated as a tool - most applications were falling into the category of "knots in physics". Now, physicists become interested in the knots themselves. As a result a new field of research, "the physics of knots", is opened. My work certainly belongs to the second category. 


\section{Acknowledgments}

This work was performed under the KBN project: 8T11F01214.I thank A. Stasiak and V. Katritch for numerous discussions. I thank the Herbette Foundation for supporting my stays in Lausanne.

\section{References}

[1] For an introduction to the theory of knots see e. g. The knot book, Collin. C. Adams, W. H. Freemann and Company, New York 1994.

[2] J. H. Przytycki, Wiadomości matematyczne, XXXI, 1 (1995).

[3] Explanantion of the origin of the integral can be found in: H. K. Moffat and R. L. Ricca, Proc. R. Soc. Lond., A439, 411 (1992).

[4] The term writhing number was introduced by F. B. Fuller, Proc. Nat. Acad. Sci. USA, 68, 815 (1971). See also Ref. 2.

[5] V. Katritch, J. Bednar, J. Michoud, R. G. Scherein, J. Dubochet and A. Stasiak, Nature, 384. 142 (1996).

[6] P. Pierański, Pro Dialog 5, 111 (1996).

[7] P. Pierański, In search of ideal knots, in IdeaI Knots, eds. A. Stasiak, V. Katritch and L. H. Kauffman, World Scientific, Singapore 1998.

[8] H. K. Moffatt, Nature, 384, 114 (1996).

[9] V. Katritch, W. K. Olson, P. Pierański, J. Dubochet and A. Stasiak, Nature, 388, 148 (1997). 